\title{
O impacto do coronavírus em pacientes cardiopatas
}

\author{
The impact of the coronavirus on patients with heart diseases \\ El impacto del coronavirus en pacientes con enfermedades cardíacas
}

Recebido: 01/04/2021 | Revisado: 11/04/2021 | Aceito: 12/04/2021 | Publicado: 25/04/2021

Layane Estefany Siqueira dos Santos
ORCID: https://orcid.org/0000-0002-8285-2690
Universidade Tiradentes, Brasil
E-mail: layanesiqueira20@gmail.com
Lucas Siqueira dos Santos
ORCID: https://orcid.org/0000-0001-5142-6931
Universidade Tiradentes, Brasil
E-mail: lucascrf648@gmail.com

\begin{abstract}
Resumo
Este estudo objetivou identificar quais são os impactos do coronavírus em pacientes cardiopatas. Trata-se de uma pesquisa de revisão integrativa de literatura. A busca dos artigos científicos foi realizada nas seguintes bases de dados: Biblioteca Virtual em Saúde (BVS), Literatura Latino-Americana e do Caribe em Ciências da Saúde (LILACS), Scientific Eletronic Library Online (SciELO) e PubMed. Foram selecionados 7 artigos para compor o atual estudo entre 2020 - 2021, posteriormente foram elaboradas discussões acerca dos estudos. Os manuscritos corroboram que os pacientes cardiopatas têm maiores riscos de desenvolverem a forma grave da COVID-19 e que muitas vezes esses pacientes acabam com lesões cardíacas após sua recuperação. Conclui-se que a contaminação pela COVID-19 agrava os danos ao coração e os cardiopatas facilmente desenvolvem a forma grave da doença.
\end{abstract}

Palavras-chave: Infecções por Coronavírus; Pandemia; COVID-19; Doenças cardiovasculares.

\begin{abstract}
This study aimed to identify what are the impacts of coronavirus on cardiac patients. This is an integrative literature review research. The search for scientific articles was carried out in the following databases: Virtual Health Library (VHL), Latin American and Caribbean Literature in Health Sciences (LILACS), Scientific Electronic Library Online (SciELO) and PubMed. 7 articles were selected to compose the current study between 2020 - 2021, later discussions about the studies were elaborated. The manuscripts corroborate that cardiac patients have a higher risk of developing the severe form of COVID-19 and that these patients often end up with cardiac injuries after their recovery. It is concluded that contamination by COVID-19 aggravates damage to the heart and cardiac patients easily develop the severe form of the disease.
\end{abstract}

Keywords: Coronavirus infections; Pandemic; COVID-19; Cardiovascular diseases.

\section{Resumen}

Este estudio tuvo como objetivo identificar cuáles son los impactos del coronavirus en los pacientes cardíacos. Esta es una investigación de revisión de literatura integradora. La búsqueda de artículos científicos se realizó en las siguientes bases de datos: Biblioteca Virtual en Salud (BVS), Literatura Latinoamericana y del Caribe en Ciencias de la Salud (LILACS), Biblioteca Electrónica Científica en Línea (SciELO) y PubMed. Se seleccionaron 7 artículos para componer el estudio actual entre 2020 - 2021, posteriormente se elaboraron discusiones sobre los estudios. Los manuscritos corroboran que los pacientes cardíacos tienen un mayor riesgo de desarrollar la forma grave de COVID19 y que estos pacientes a menudo terminan con lesiones cardíacas después de su recuperación. Se concluye que la contaminación por COVID-19 agrava el daño al corazón y los pacientes cardíacos desarrollan fácilmente la forma severa de la enfermedad.

Palabras clave: Infecciones por coronavirus; Pandemia; COVID-19; Enfermedades cardiovasculares.

\section{Introdução}

Em dezembro de 2019, a China comunicou à Organização Mundial da Saúde (OMS) sobre um surto de doença respiratória aguda grave que se concentrou na cidade de Wuhan (Opas, 2020). Revelou-se assim um novo vírus que foi nomeado como coronavírus (2019-nCoV) e também chamado SARS-CoV-2. A situação levou a OMS a declarar a situação como sendo de importância Internacional (Nepal, et al., 2020; Rafael, et al., 2020). 
A COVID-19 é uma doença respiratória causada por um novo tipo de coronavírus (SARS-CoV-2), que já acometeu milhares de pessoas em todo mundo. Os sinais e sintomas mais comuns causados pelo coronavírus incluem febre, fadiga, coriza, tosse seca, sangramento nasal, mialgia, dispneia, insuficiência renal, linfopenia grave e tempo prolongado de protrombina são também as características mais comuns. O diagnóstico dos casos sintomáticos é confirmado por meio da pesquisa do vírus através da coleta de um swab nasal (Li, et al., 2020; Ramos, et al., 2020).

Sabe-se que a doença afeta mais pessoas adultas com idade acima de 60 anos com ou sem comorbidades, apresentando-se de forma mais grave em quem possui doenças crônicas (entre elas as doenças cardiovasculares). Os cardiologistas têm observado que os pacientes cardiopatas contaminados pelo coronavírus têm apresentado complicações em seu quadro clínico se comparado com os demais clientes (Gallasch, et al., 2020; Lin, et al., 2020).

As complicações cardíacas causadas por essa doença têm chamado a atenção dos clínicos. Um estudo realizado no Hospital San Raffaele, em Milão/Itália, hospital de referência para complicações cardiovasculares da COVID-19, foi analisado que 16,7\% dos pacientes contaminados desenvolveram arritmias e 7,2\% apresentaram lesão cardíaca aguda. Foram coletadas enzimas cardíacas (BNP, troponina, CK-MB) de todos os pacientes o que detectou sinal de comprometimento cardíaco (Strabelli, 2020)

Além disso, surge a preocupação se os cardiopatas estão em maior risco para desenvolver complicações e se novas infecções pelo COVID-19 têm impacto no sistema cardiovascular. De acordo com pesquisas realizadas na cidade de Chen, localizada na China, mostraram que pacientes com presença de doenças cardíacas têm maiores riscos de morbimortalidade quando contaminados pelo coronavírus ( $\mathrm{Li}$, et al., 2020).

Como medida de prevenção é necessário que os cardiopatas utilizem medidas de precaução, como o isolamento social. Os medicamentos de rotina devem continuar tomando segundo recomendações do cardiologista que o acompanha. Deve-se também manter atualizada a carteira vacinal principalmente no que diz respeito à vacina contra a Influenza (Gallasch, et al., 2020). Com isso, o objetivo deste estudo foi identificar quais são os impactos do coronavírus em pacientes cardiopatas.

\section{Metodologia}

Trata-se de uma pesquisa de revisão integrativa de literatura. Este tipo de pesquisa é definido como método que permite analisar as informações de um determinado assunto por meio da síntese dos resultados de estudos anteriores. As bases de dados utilizadas para coleta dos dados foram: Biblioteca Virtual em Saúde (BVS), Literatura Latino-Americana e do Caribe em Ciências da Saúde (LILACS), Scientific Eletronic Library Online (SciELO) e PubMed.

Foram empregados os seguintes descritores para elaboração do estudo: Infecções por Coronavírus; Pandemias; COVID-19; Doenças cardiovasculares, de acordo com os Descritores em Ciências da Saúde (DECS). Foi utilizado combinações dos descritores com auxílio do operador lógico booleano "AND". Com base nos resultados de busca, foi procedida a leitura de todos os títulos, seguida da leitura dos resumos das produções científicas.

Os critérios de inclusão foram estudos publicados no período entre o ano de 2020-2021, em idioma português e inglês, com o texto completo, disponível on-line gratuitamente para leitura e/ou download. Os artigos selecionados foram analisados na íntegra, avaliando as discussões referentes ao impacto do coronavírus em pacientes cardiopatas.

Após aplicados os critérios de elegibilidade acima descritos, excluídas as duplicidades e lidos os resumos com o objetivo de identificar aqueles que versavam adequadamente sobre o tema, foram selecionados 94 artigos. Dessa forma, após a leitura na íntegra dos mesmos, foram escolhidos sete artigos conforme o fluxograma a seguir (Figura 1). 
Figura 1. Fluxograma das etapas de coleta de dados.

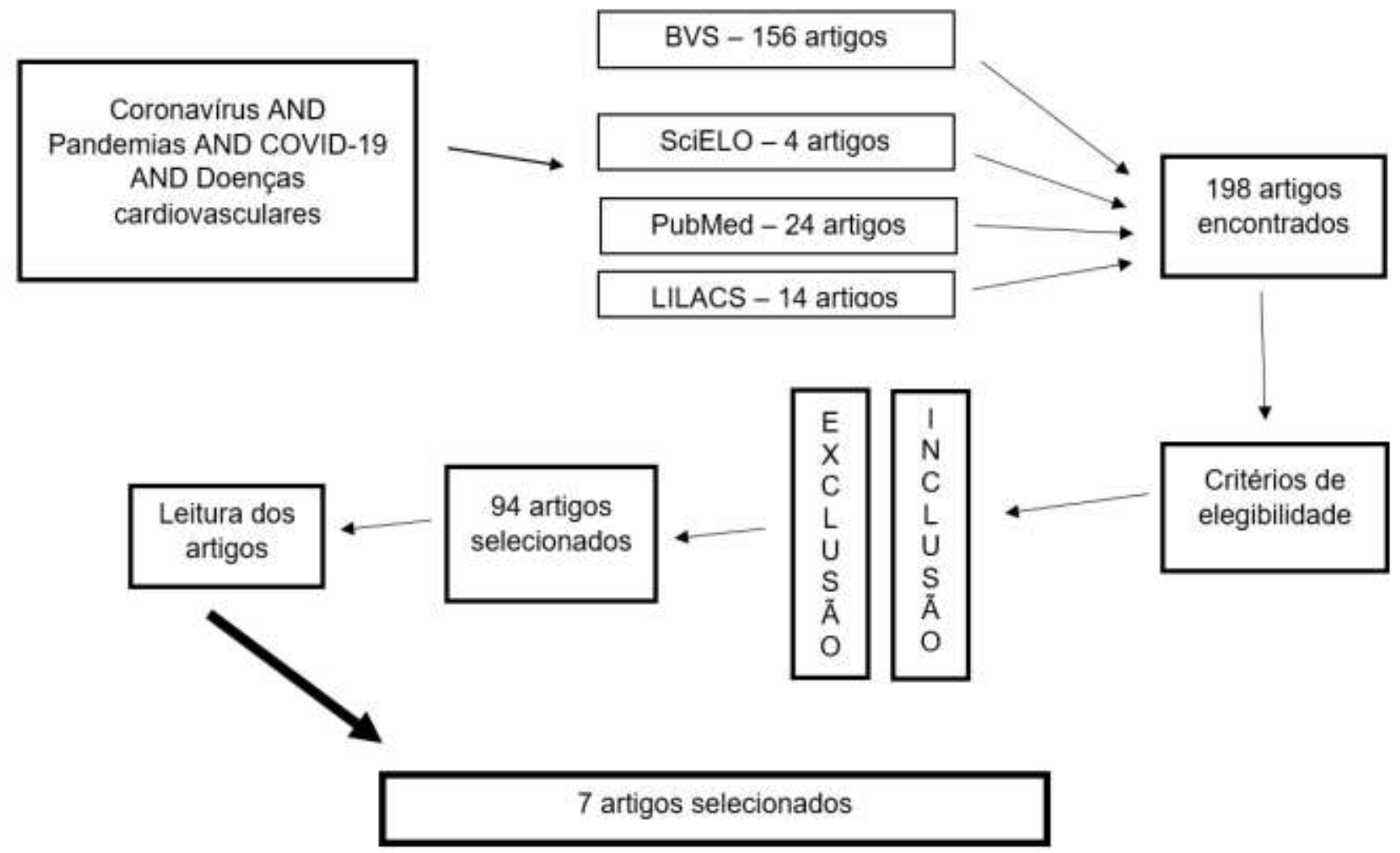

Fonte: Autores.

Por se tratar de dados secundários, não foi necessária a submissão ao Comitê de Ética em Pesquisa. Entretanto, os dados obtidos por meio dessa seguiram princípios éticos, segundo a Lei dos Direitos Autorais número 12.853/2013. As informações contidas neste estudo foram citadas de forma fiel, conforme bibliografia selecionada e preconizada pela Associação Brasileira de Normas Técnicas (ABNT) NBR 6023 e NBR 10520.

\section{Resultados}

A partir da análise dos artigos e, após estabelecer os critérios de elegibilidade, os estudos foram organizados em uma tabela, cuja finalidade é facilitar a visualização dos pontos que se relacionam com a temática em questão (Tabela 1). 
Tabela 1. Descrição dos artigos selecionados de acordo com título, autor, ano, tipo de estudo, periódico, objetivo e desfecho.

\begin{tabular}{|c|c|c|c|c|c|}
\hline TÍTULO & $\begin{array}{l}\text { AUTOR / } \\
\text { ANO }\end{array}$ & $\begin{array}{l}\text { TIPO DE } \\
\text { ESTUDO }\end{array}$ & PERIÓDICO & OBJETIVO & DESFECHO \\
\hline $\begin{array}{c}\text { Prevalence } \\
\text { and impact } \\
\text { of cardiovascular } \\
\text { metabolic diseases on } \\
\text { COVID-19 in China. }\end{array}$ & $\begin{array}{l}\text { Li, et al., } \\
2020\end{array}$ & $\begin{array}{l}\text { Revisões } \\
\text { sistemáticas e } \\
\text { meta-análises } \\
\text { de dados }\end{array}$ & $\begin{array}{l}\text { Revista Clínica } \\
\text { em cardiologia. }\end{array}$ & $\begin{array}{l}\text { Determinar a associação } \\
\text { de doenças metabólicas } \\
\text { cardiovasculares com o } \\
\text { desenvolvimento de } \\
\text { COVID-19 }\end{array}$ & $\begin{array}{l}\text { A COVID-19 pode causar } \\
\text { danos ao coração. }\end{array}$ \\
\hline $\begin{array}{l}\text { COVID-19 e o } \\
\text { Coração }\end{array}$ & $\begin{array}{l}\text { Strabelli \& } \\
\text { Uip, } 2020\end{array}$ & $\begin{array}{l}\text { Estudo } \\
\text { retrospectivo }\end{array}$ & $\begin{array}{l}\text { Arq. Bras. } \\
\text { Cardiol. }\end{array}$ & $\begin{array}{l}\text { Associar a relacão da } \\
\text { COVID-19 ao coração }\end{array}$ & $\begin{array}{l}\text { Os cardiopatas têm } \\
\text { maiores riscos de morte se } \\
\text { adquirirem COVID-19 se } \\
\text { comparados com os } \\
\text { demais pacientes. }\end{array}$ \\
\hline $\begin{array}{l}\text { Implicações de } \\
\text { doenças } \\
\text { cardiovasculares na } \\
\text { evolução de } \\
\text { prognóstico } \\
\text { em pacientes com } \\
\text { COVID-19 }\end{array}$ & $\begin{array}{l}\text { Cavalcante, et } \\
\text { al., } 2021\end{array}$ & $\begin{array}{c}\text { Revisão } \\
\text { literária } \\
\text { integrativa }\end{array}$ & $\begin{array}{c}\text { Revista } \\
\text { Eletrônica } \\
\text { Acervo } \\
\text { Saúde/Electronic } \\
\text { Journal } \\
\text { Collection Health }\end{array}$ & $\begin{array}{l}\text { Retratar uma relação entre } \\
\text { comorbidades, } \\
\text { especialmente } \\
\text { cardiovasculares, e os } \\
\text { desfechos da COVID-19 }\end{array}$ & $\begin{array}{c}\text { Alguns pacientes } \\
\text { desenvolveram lesões } \\
\text { cardíacas após contrair o } \\
\text { Coronavírus. }\end{array}$ \\
\hline $\begin{array}{c}\text { Impact of } \\
\text { cardiovascular } \\
\text { disease and risk } \\
\text { factors on fatal } \\
\text { outcomes in patients } \\
\text { with COVID-19 } \\
\text { according to age: a } \\
\text { systematic review } \\
\text { and meta-analysis. }\end{array}$ & $\begin{array}{l}\text { Bae, et al., } \\
2021\end{array}$ & $\begin{array}{l}\text { Revisão } \\
\text { sistemática e } \\
\text { metanálise }\end{array}$ & Heart & $\begin{array}{l}\text { Investigar o impacto da } \\
\text { doença cardiovascular } \\
(\mathrm{DCV}) \text { e seus fatores de } \\
\text { risco em desfechos fatais } \\
\text { de acordo com a idade em } \\
\text { pacientes com COVID-19 }\end{array}$ & $\begin{array}{l}\text { Pacientes, de todas as } \\
\text { idades, com hipertensão e } \\
\text { diabetes, geralmente } \\
\text { evoluem rapidamente para } \\
\text { desfechos fatais quando } \\
\text { contaminados pela } \\
\text { COVID-19. }\end{array}$ \\
\hline $\begin{array}{c}\text { Cardiovascular } \\
\text { implications of fatal } \\
\text { outcomes of patients } \\
\text { with coronavírus } \\
\text { disease 2019 (covid- } \\
19 \text { ) }\end{array}$ & $\begin{array}{l}\text { Guo, et al., } \\
2020\end{array}$ & $\begin{array}{l}\text { Estudo } \\
\text { observacional } \\
\text { retrospectivo }\end{array}$ & $\begin{array}{c}\text { JAMA } \\
\text { cardiology }\end{array}$ & $\begin{array}{l}\text { Avaliar a associação de } \\
\text { doença cardiovascular de } \\
\text { base (DCV) e lesão } \\
\text { miocárdica com desfechos } \\
\text { fatais em pacientes com } \\
\text { COVID-19. }\end{array}$ & $\begin{array}{l}\text { Lesão miocárdica tem um } \\
\text { vínculo significativo com } \\
\text { resultados fatais de } \\
\text { COVID-19. }\end{array}$ \\
\hline $\begin{array}{c}\text { Cardiovascular } \\
\text { disease and COVID- } \\
19\end{array}$ & $\begin{array}{l}\text { Bansal, et al., } \\
2020\end{array}$ & $\begin{array}{l}\text { Revisão } \\
\text { bibliográfica }\end{array}$ & $\begin{array}{c}\text { Diabetes \& } \\
\text { Metabolic } \\
\text { Syndrome: } \\
\text { Clinical Research } \\
\text { \& Reviews }\end{array}$ & $\begin{array}{l}\text { Avaliar a associação de } \\
\text { doença cardiovascular de } \\
\text { base (DCV) e lesão } \\
\text { miocárdica com desfechos } \\
\text { fatais em pacientes com } \\
\text { COVID-19. }\end{array}$ & $\begin{array}{l}\text { Os trabalhos descreveram } \\
\text { brevemente as alterações } \\
\text { cardiovasculares, todavia } \\
\text { foi possível identificar que } \\
\text { a Covid-19 piora o quadro } \\
\text { clínico dos pacientes } \\
\text { cardiopatas. }\end{array}$ \\
\hline $\begin{array}{l}\text { Cardiac involvement } \\
\text { in COVID-19 } \\
\text { patients: Risk factors, } \\
\text { predictors, and } \\
\text { complications: A } \\
\text { review }\end{array}$ & $\begin{array}{l}\text { Aghagoli, et } \\
\text { al., } 2020\end{array}$ & $\begin{array}{l}\text { Revisão de } \\
\text { literatura }\end{array}$ & $\begin{array}{c}\text { Journal of } \\
\text { cardiac surgery }\end{array}$ & $\begin{array}{l}\text { Descrever as implicações } \\
\text { cardíacas do COVID-19. }\end{array}$ & $\begin{array}{c}\text { Mais estudos se faz } \\
\text { necessário para afirmar } \\
\text { com efetividade a } \\
\text { associação da COVID-19 } \\
\text { com o agravamento do } \\
\text { quadro clínico dos } \\
\text { cardiopatas. }\end{array}$ \\
\hline
\end{tabular}

Fonte: Autores.

Em relação aos resultados, os manuscritos corroboram que os pacientes cardiopatas têm maiores riscos de desenvolverem a forma grave da Covid-19 e que muitas vezes esses pacientes acabam com lesões cardíacas após sua recuperação. 


\section{Discussão}

Bae, et al. (2021) afirmaram que os grupos com maiores riscos para COVID-19 são os adultos acima de 60 anos que possuem doenças crônicas como: hipertensão arterial sistêmica, câncer, diabetes mellitus, doença cardiovascular crônica, doença pulmonar crônica, doença renal crônica e imunossuprimidos. O estudo realizado por Li, et al. (2020) na cidade de Chen, na China, está aliado com o estudo de Bae, et al. (2021), pois demonstrou que os indivíduos infectados com a COVID19 possuíam comorbidades como hipertensão, diabetes e doença cardio-cerebrovascular. No estudo, $40 \%$ dos pacientes tinham doença cardio-cerebrovascular e $20 \%$ tinham diabetes.

Segundo Strabelli e Uip (2020), a incidência de lesão miocárdica é muito maior em pacientes graves, cerca de 13 vezes mais em comparação a pacientes não graves. Além disso, o estudo demonstrou que pacientes com COVID-19 associados a angina instável apresentam insuficiência cardíaca, pneumonia grave, levando à deterioração de órgãos. De acordo com a apresentação clínica e os dados laboratoriais da doença, pode-se especular que a infecção pelo coronavírus pode afetar o sistema cardiovascular através de múltiplos mecanismos.

O estudo realizado por Cavalcante, et al. (2021) afirmaram que a COVID-19 causa possível sequela no tecido miocárdico. A hipoxemia causada pela pneumonia causa alteração na troca gasosa, e a depressão do sistema respiratório pode ser uma importante razão das cardiopatias. A hipoxemia, que reduz significativamente o suprimento de energia pelo metabolismo celular, leva a lesões e apoptose dos cardiomiócitos. Essas sequelas cardíacas acabam afetando diretamente na qualidade de vida desses pacientes pós COVID-19.

O estudo de Guo, et al. (2020) corrobora com o estudo de $\mathrm{Li}$, et al. (2020), pois ambos confirmaram a presença de lesões miocárdicas, demonstradas pela elevação dos níveis de Troponina-T (TnT), levando a um aumento da mortalidade nesses pacientes em comparação com os demais, com mortalidade de 69,44\%, com menor chances de sobrevida. Além disso, os pacientes cardiopatas tinham vários graus de hipoxemia e precisavam de oxigenoterapia.

Aghagoli, et al., (2020) afirmaram em seu estudo que os pacientes com comorbidades são mais propensos a adquirirem doenças cardíacas quando contaminados pelo COVID-19. As arritmias cardíacas comprometem as células do miocárdio, causando inflamação sistêmica, levando a formação de placas coronarianas levando a hipóxia e até mesmo ao infarto agudo do miocárdio. Bansal, et al. (2020) revelaram que a infecção pode ocasionar um desequilíbrio da resposta imunológica, contribuindo assim para lesões no miocárdio, devido a redução do fluxo sanguíneo coronário, diminuição do aporte de oxigênio, formações de placas coronarianas assim como micro trombogênese.

Gallasch, et al. (2020) e Aghagoli, et al. (2020) falaram da importância dos cardiopatas manter certo grau de isolamento em domicílio, evitando-se frequentar locais de aglomeração, manter uma alimentação saudável, manter as rotinas de exercícios diários, manter sempre uma distância de 1 metro de outra pessoa, evitando abraços, beijos e apertos de mão. Deve-se estar atento aos cuidadores, evitando ao máximo o contato com pessoas com sintomas gripais, deve-se lavar muito bem as mãos cobrindo nariz, boca e braço ao tossir e espirrar. A COVID-19 em um cardiopata pode ter uma evolução muito rápida e de altíssima gravidade. Além disso, é recomendado reforçar a necessidade de manter atualizada a carteira de vacinação desses cardiopatas.

No presente estudo, observou-se às diversas complicações que um cardiopata pode desenvolver ao contrair a COVID19. Todavia, é importante ressaltar que a temática ainda é nova e requer estudos mais aprofundados, pois foi possível visualizar uma limitação dos estudos em relação aos reais impactos que a COVID-19 pode causar no paciente cardiopata ao longo do tempo, mesmo após a cura. 


\section{Conclusão}

Estudos vêm mostrando que as comorbidades cardiovasculares tornaram os pacientes mais propícios a serem contaminados pela COVID-19, levando essas pessoas a terem um diagnóstico mais grave em relação à doença. As doenças cardíacas são condições comuns entre os pacientes hospitalizados com a COVID-19 e estão associadas a um risco maior de mortalidade hospitalar. O coronavírus ocasiona danos no sistema cardiovascular causando inflamação dos vasos sanguíneos e o aumento de trombos. É importante destacar que, a contaminação pela COVID-19 agrava os danos ao coração. Mesmo que o mecanismo do problema cardíaco precise ser estudado em cada caso, os resultados apresentados nesse estudo revelam a necessidade de considerar essas complicações na gestão da doença.

Como sugestões para trabalhos futuros, se faz necessário conduzir estudos para o aprofundamento dos impactos da COVID-19 em cardiopatas, focando nos sinais, sintomas e qualidade de vida pós-COVID-19. Além disso, é essencial que realizem pesquisas para descrever o perfil epidemiológico e socioeconômico desses pacientes.

\section{Referências}

Aghagoli, G., Gallo Marin, B., Soliman, L. B., \& Sellke, F. W. (2020). Cardiac involvement in COVID-19 patients: Risk factors, predictors, and complications: A review. Journal of cardiac surgery, 35(6), 1302-1305.

Bae, S., Kim, S. R., Kim, M. N., Shim, W. J., \& Park, S. M. (2021). Impact of cardiovascular disease and risk factors on fatal outcomes in patients with COVID-19 according to age: a systematic review and meta-analysis. Heart, 107(5), 373-380.

Bansal, M. (2020). Cardiovascular disease and COVID-19. Diabetes \& Metabolic Syndrome: Clinical Research \& Reviews, 14(3), 247-250.

Cavalcante, I. S., de Lima, C. V. B. Q., Mendes, J. P. S., Barbosa, J. V. C., Neto, O. J. F., Rodrigues, P. F., \& Beltrão, R. P. L. (2021). Implicações de doenças cardiovasculares na evolução de prognóstico em pacientes com covid-19. Revista Eletrônica Acervo Saúde, 13(1), e5292-e5292.

Gallasch, C. H., da Cunha, M. L., de Souza Pereira, L. A., \& Silva-Junior, J. S. (2020). Prevenção relacionada à exposição ocupacional do profissional de saúde no cenário de COVID-19 [Prevention related to the occupational exposure of health professionals workers in the COVID-19 scenario][Prevención relacionada cone la exposición ocupacional de profesionales de la salud en el escenario COVID-19]. Revista Enfermagem, UERJ, $28,49596$.

Guo, T., Fan, Y., Chen, M., Wu, X., Zhang, L., He, T., \& Lu, Z. (2020). Cardiovascular implications of fatal outcomes of patients with coronavirus disease 2019 (COVID-19). JAMA cardiology, 5(7), 811-818.

Li, B., Yang, J., Zhao, F., Zhi, L., Wang, X., Liu, L., \& Zhao, Y. (2020). Prevalence and impact of cardiovascular metabolic diseases on COVID-19 in China. Clinical Research in Cardiology, 109(5), 531-538.

Lin, C., Arevalo, Y. A., Nanavati, H. D., \& Lin, D. M. (2020). Racial differences and an increased systemic inflammatory response are seen in patients with COVID -19 and ischemic stroke. Brain, Behavior, \& Immunity - Health, 8 (July), 100137. DOI: doi.org/10.1016/j.bbih.2020.100137

Mendes, K. D. S., Silveira, R. C. D. C. P., \& Galvão, C. M. (2008). Revisão integrativa: método de pesquisa para a incorporação de evidências na saúde e na enfermagem. Texto \& contexto-enfermagem, 17(4), 758-764.

Nepal, G., Rehrig, J. H., Shrestha, G. S., Shing, Y. K., Yadav, J. K., Ojha, R., Pokhrel, G., Tu, Z. L., \& Huang, D. Y. (2020). Neurological manifestations of COVID-19: A systematic review. Critical Care, 24(1), 1-11.

Opas. (2020). Organização Pan - Americana da Saúde. Folha informativa - COVID - 19 (doença causada pelo novo coronavírus). https://www.paho.org/bra/index.php?option=com_content \&view=article \&id=6101:covid19\&Itemid=875

Rafael, R. D. M. R., Neto, M., de Carvalho, M. M. B., David, H. M. S. L., Acioli, S., \& de Araujo Faria, M. G. (2020). Epidemiologia, políticas públicas e pandemia de Covid-19: o que esperar no Brasil? [Epidemiology, public policies and Covid-19 pandemics in Brazil: what can we expect?] [Epidemiologia, políticas públicas y la pandémia de Covid-19 en Brasil: que podemos esperar?]. Revista enfermagem, UERJ, $28,49570$.

Ramos, R. M. (2020). Análise do perfil epidemiológico dos óbitos por COVID-19 em Santa Catarina durante a pandemia de coronavírus até a 33ª semana epidemiológica do ano de 2020. TCC (graduação) - Universidade Federal de Santa Catarina. Centro de Ciências da Saúde. Medicina.

Strabelli, T. M. V., \& Uip, D. E. (2020). COVID-19 e o Coração. Arquivos Brasileiros de Cardiologia, 114(4), 598-600. 